\title{
Efficiency of health facilities providing antiretroviral treatment services in Botswana
}

\author{
Carlos Avila ${ }^{1}$, Wu Zeng ${ }^{2}$, Chris Cintron ${ }^{3}$ \\ ${ }^{1}$ Palladium, International Health Practice, London, UK; ${ }^{2}$ Department of International Health, School of Nursing \& Health Studies, Georgetown \\ University, Washington, DC, USA; ${ }^{3}$ Palladium, International Health Practice, Washington, DC, USA \\ Contributions: (I) Conception and design: C Avila, W Zeng; (II) Administrative support: None; (III) Provision of study materials or patients: None; (IV) \\ Collection and assembly of data: C Cintron, C Avila; (V) Data analysis and interpretation: W Zeng, C Avila; (VI) Manuscript writing: All authors; (VII) \\ Final approval of manuscript: All authors. \\ Correspondence to: Carlos Avila. Director of Health Financing, International Health Practice, EMEA, Tumberry House, 100 Bunhill Row, London \\ EC1Y 8NA, UK. Email: carlos.avila@thepalladiumgroup.com.
}

Background: Botswana is implementing an ambitious universal health coverage agenda and successfully expanding antiretroviral treatment to nearly 380,000 people living with human immunodeficiency virus (HIV). However, the country needs to critically assess its efficient use of all available resources to sustain gains and continue progress to attain the targets and vision for ending acquired immune deficiency syndrome (AIDS) as a public health threat by 2030. The objective of this study was to measure costs and efficiency of antiretroviral therapy (ART) provision in Botswana's public facilities, and to identify opportunities for potential savings in order to expand ART coverage.

Methods: We applied an activity-based costing approach to a random sample of 2,393 adults receiving ART outpatient services in 120 facilities in Botswana. All costs are reported in 2017 US\$. We defined efficiency as the amount of resources that is put into the delivery of healthcare in relation to the amount of people receiving services. Employing a health systems perspective, the production of antiretroviral healthcare services includes three major inputs: labor, antiretroviral drugs and laboratory tests. The production unit was defined as an ART clinical unit running in a hospital, primary health care (PHC) clinic, or health post. Data envelopment analysis (DEA) was used to identify the most efficient facilities and regression analysis to identify factors associated with technical efficiency.

Results: The average unit cost of ART care was US\$297 per adult per year across levels of care (US\$379 at health posts, US\$324 at hospitals, and US\$266 at PHC clinics). Patients at all levels of care received almost quarterly clinical check-ups and were monitored with at least one viral load and one CD4 test per year. On average, PHC clinics serve 400 patients per clinical full-time equivalent (FTE) staff compared to 341 in hospitals and 77 in health posts. DEA resulted in efficiency scores for health posts of $78.3 \%$, as compared to $76.0 \%$ for clinics and $72.4 \%$ for hospitals. The overall performance of health facilities in providing ART in Botswana is about $75 \%$, which suggests that improving efficiency would result in $25 \%$ savings if the provision of services is optimized. A regression analysis showed that a higher average number of general laboratory tests per patient and a higher proportion of clinical staff to total staff were associated with a significant decrease in efficiency.

Conclusions: PHC clinics achieved lower unit costs by providing services to many more patients, suggesting economies of scale. Treatment costs are higher in health posts, mainly attributable to more staff treating fewer patients, suggesting diminishing marginal returns as ART coverage expands into a low patient volume in rural areas. Our findings demonstrate there is room to increase outputs (number of patients on ART) under the current set of inputs (physical or financial) across all levels of care. Future reduction in antiretroviral purchasing costs, updating guidelines to standardize and reduce excessive laboratory testing, task sharing and implementing differentiated models of care are potential interventions to reduce average costs and improve efficiency in provision of ART services. 
Keywords: Costing; antiretroviral treatment; efficiency; Botswana

Received: 02 June 2020; Accepted: 27 October 2020; Published: 25 December 2020.

doi: 10.21037/jhmhp-20-75

View this article at: http://dx.doi.org/10.21037/jhmhp-20-75

\section{Introduction}

In response to an adult human immunodeficiency virus (HIV) prevalence of $20.3 \%$ and approximately 380,000 of its citizens living with HIV (1), Botswana has made HIV/ acquired immune deficiency syndrome (AIDS) care a health priority. Botswana and its development partners have launched strong efforts to support programs for preventing mother-to-child transmission of HIV and providing treatment to those who need it. Botswana's efforts to treat and prevent HIV/AIDS have made significant progressBotswana is already close to reaching the ambitious 90-9090 UNAIDS targets (2); $91 \%$ of people living with HIV know their status, $83 \%$ of people who know their HIV status are on antiretroviral therapy (ART), and $81 \%$ have reached virologic suppression (1). The country has been successful in providing free universal access of ART to the HIV-affected population. Treatment programs have shown significant impact; from 2010 to 2018, antiretroviral (ARV) coverage increased from $47 \%$ to $83 \%$, HIV incidence declined by $36 \%$, and AIDS-related deaths were reduced by $33 \%$ (3).

Despite widespread coverage of HIV programs, the country still ranks fourth in terms of global HIV prevalence after South Africa, Lesotho and Eswatini. Mortality due to HIV has declined but the country is struggling to meet targets to reduce new HIV cases, and there has been a decline in adherence to treatment among the HIV-affected population (3). Thus, continued support and commitment to ensuring universal access to treatment remain crucial to further improve health outcomes for HIV patients. Botswana's National Strategic Framework for HIV and AIDS reinforces its strong commitment towards ending the AIDS epidemic by 2030 (1). It is expected that this new strategy will increase the number of HIV patients receiving ART with a corresponding increase in the cost to the HIV/ AIDS programs.

In response to these needs, there is a strong national commitment to increasing ownership of HIV prevention, care, and treatment efforts. With donor support decreasing and scope of treatment expanding through Treat All, it is critical that the Government of Botswana effectively use domestic resources to preserve the gains from HIV/ AIDS programming while continuing progress toward national goals. These increasing resources needs require improvements in allocation of available resources and gains in efficient use of inputs, including reduction of waste and overall increase in health facility productivity.

The high coverage of ART demands adequate financing to sustain it and US\$376 million were allocated to finance HIV/AIDS programmes in the 2018/19 budget (4); this amount is expected to rise year after year towards the goal to ending the AIDS epidemic by 2030. Financial sustainability is critical to continue providing universal access to the highly successful, cost-free ART treatment programme. The HIV/AIDS epidemic poses a great burden to the government to finance ART and overall health, and about half of Botswana's health expenditure was allocated to HIV. Given the tight budget that the Government of Botswana has and the potential decline of donor funding for HIV/AIDS services, it is important to ensure the existing resources are used efficiently.

Efficiency improvement has great potential to improve a country's fiscal space for health. At the global level, Zeng et al. estimates potential savings of $50 \%$ of funding for HIV/AIDS derived from gains in efficiency (5). At the country level, the variation is smaller, but remains nonnegligible (6). In Botswana, challenges to provide ART have been reported, such as human resource constraints, lack of essential technical skills to provide services, and shortage of anti-retroviral drugs. All these challenges suggest that the ART services are not provided optimally and there is room for further improvement.

In this study, we evaluate the performance status of public health facilities in providing ART among HIV/AIDS populations, and then explore factors that may affect the performance of providing ART services, in order to improve the efficiency of health facilities by addressing potential barriers. We present the following article in accordance with the MDAR reporting checklist (available at http:// dx.doi.org/10.21037/jhmhp-20-75). 


\section{Methods}

We used the conventional two-step approach to conduct this efficiency study. The first step was to use a data envelopment analysis (DEA) to examine the efficiency of health facilities in providing ART services using a set of inputs and outputs. The second step was to use a regression model to investigate determinants of the efficiency, once efficiency scores were estimated from the first step $(7,8)$. This study did not involve individual patients; however, it was submitted and approved by the Health Research and Development Committee (HRDC), Ministry of Health, Botswana.

\section{Sample selection}

The sampling unit for this study was the health facility, and the study population was public hospitals, clinics and health posts that offered ART services. In Botswana, there were 29 public hospitals, 210 public clinics, and 45 public health posts eligible for the study, constituting the study population. All the 29 public district hospitals were selected in the analysis. Public clinics were first sorted by district, and a sampling approach of probability proportional to size (PPS) of health facilities was used to select sample facilities to be included in the study. The size was measured in terms of the number of patients receiving ARV therapy in each facility; 73 public clinics were drawn from 210 public clinics and 18 public health posts out of a total of 45 health posts were selected for this study.

To estimates costs, records for twenty adult patients who received ART were randomly selected in each facility. The patient records collected information regarding ARV regiments, frequency of lab tests and patient visits, which were used as inputs to estimate the level of efficiency of health facilities in providing ambulatory ART services.

\section{Inputs and outputs used for the DEA}

We applied DEA to evaluate the efficiency of health facilities in providing ART services. This method allows for modelling multiple inputs and outputs to generate an efficiency score for each facility. Consistent to the categories of resources used to provide ART services, we defined the inputs for the DEA as: (I) the number of fulltime equivalent (FTE) clinical staff providing ART services; (II) the number of FTE non-clinical staff supporting ART services; and (III) combined costs of ARV, lab tests and medical supplies for ART treatment. The two outputs for DEA were: (I) the number of HIV patients on first-line ARV treatment; and (II) the number of HIV patients on second line ARV treatment.

\section{Information on human resources providing ART services}

Data on FTE clinical staff and FTE non-clinical staff was collected through a facility survey. Managers as well as personnel providing HIV services in health facilities were asked about their time allocation for ART and other services, and then this information was aggregated as FTE at the health facility level.

\section{Cost of ARV, lab tests, and HIV supplies}

Data on volume of ARV and ART schemes were obtained from patient records. The prices of ARV came from Central Medical Store (CMS) that procured ARV centrally, supplemented from other sources if CMS did not have the price information on some ARV drugs. Similarly, utilization of lab tests came from patient records, and the unit cost of lab test was derived from secondary data. The costs of HIV supplies were estimated from two HIV clinics with the most complete cost information on HIV supplies. All the costs are reported in 2017 US\$.

\section{Output data of adult ART patients at each facility}

Data on the total number of patients receiving treatment at HIV clinics were collected at the facility level and compared to the centrally reported database. When the information at the facility level was missing or incomplete, we included aggregated patients on ART included in the national database. To estimate the total number of adult ART patients per facility, pediatric patients in the relevant ART site were excluded from the total number of ART patients. Patients were specified as receiving first or second line treatment based on their ARV regimen.

\section{Contextual factors determining the efficiency}

We also analyzed a series of contextual variables that may affect the efficiency. These contextual variables focused on type of facilities, share of HIV/AIDS services available, and HIV epidemic level of the catchment areas. These contextual variables include: (I) type of facility, whether a health facility was a health post, clinic or hospital; (II) a variable indicating whether a health facility had lab or not; (III) a variable indicating whether a health facility received external funding from donors or not; (IV) a variable 
indicating whether a health facility had observation beds or not; (V) a variable indicating whether a health facility had inpatient beds or not; (VI) share of HIV staff among the total number of staff in the health facility; (VII) share of non-clinical staff working on ART to the total number of staff working on ART; and (VIII) district HIV prevalence. All data were collected from the health facility survey.

\section{Data collection}

The research team trained 16 local data collectors to administer the questionnaire forms and visit the facilities in pairs. Two data collection forms were developed: (I) the facility form collected facility-level data, including data on ART visits, human resources, and the stocks of ARVs and other supplies; (II) the patient record form collected information around number of visits over the study period, ARV regimens and dates of switch to second or third line regimens, and number of lab tests performed.

Data collection took place in two phases, the first from November 16, 2015 to December 12, 2015 and the second from April 11, 2016 to May 6, 2016. Between the two phases, the study team reviewed surveys from the first round of data collection, identifying data gaps and areas that required further follow-up. Any issues with data collection were brought to the attention of the data collectors, and, during the second phase of data collection, the data collectors filled critical gaps.

Data were entered from the paper forms into FluidSurvey, a structured data entry template, between May and June 2016. The data were then cleaned and prepared for the costing analysis. The study team used Microsoft Excel and Stata 12.0 for the data analysis. The data were stored electronically, on password-protected laptops.

Secondary data from central offices were compiled for the analysis. The CMS provided procurement prices of ARVs and of clinic and lab supplies. The Ministry of Health provided salary grades for public sector employees, the number of ART patients, and other facility variables. Additional data from secondary sources, for example, on lab test costs and inflation and exchange rates for Botswana, were also compiled.

\section{Statistical analysis}

Descriptive analyses were conducted on input and output variables, as well as contextual variables. For continuous variables, means and standard deviations were presented while the frequency was calculated for categorical variables. We estimated efficiency scores using an input-oriented DEA model. To correct for potential biases due to the datagenerating process in DEA, we adopted Simar's approach (9) to generate bias-corrected efficiency score using R. We conducted two types of DEA; first, using data from hospitals, clinics, and health post separately, we estimated three production frontiers for each type of health facility and generated efficiency scores based on these three production frontiers (separate efficiency hereafter). Next, we pooled data from all three types of health facilities in one production frontier, and generated efficiency scores based on the pooled data (pooled efficiency hereafter).

Bivariate analyses were conducted between efficiency and each of the contextual variables included in the analysis. As all contextual variables were categorical variables, analysis of variance (ANOVA) was used to analyze the efficiency differences for contextual variables with more than two categories, while $t$-tests were used for contextual variables with two categories.

To examine the impact of contextual factors on efficiency, a hierarchical linear modeling (HLM) assuming a random intercept at the district level was used to examine the impact of contextual factors on efficiency, given that the independent variables contained both facility and district level variables. The model could be expressed as:

$$
\begin{gathered}
\text { Efficiency }_{i j}=\beta_{0}+B X+\varepsilon_{i j} \\
\beta_{0}=\overline{\beta_{0}}+\beta_{i}
\end{gathered}
$$

where $i$ represents $i$ th district and $j$ represents $j$ th facility. $\beta_{0}$ is the intercept of the overall model, $\mathrm{B}$ is coefficient matrix for contextual variables, and $\varepsilon_{i j}$ is the random noise at the individual facility level. $\overline{\beta_{0}}$ is the grand intercept cross districts and $\beta_{i}$ is the random noise at the district level. The statistical analyses were conducted using $\mathrm{R}$.

\section{Results}

\section{Descriptive analysis of inputs, outputs and contextual factors}

Table 1 provides the descriptive statistics of input and outputs variables collected from the 120 facilities. On average, there were 3.92 clinical staff and 5.83 non-clinical staff providing ART related services in each health facility. The cost of ARV, lab tests and supplies were $\$ 356,444$ per facility, and this number varied substantially across facilities with the standard deviation of $\$ 332,303$. Information from 
Table 1 Description of inputs and outputs

\begin{tabular}{lccc}
\hline Variables & N & Mean & 3.25 \\
\hline Number of clinical staff & 120 & 3.92 & 5.83 \\
Number of non-clinical staff & 120 & 356,444 & 332,303 \\
Cost of ARV, lab test and supplies (US\$) & 120 & 1,293 & 1,066 \\
Number of first line patients & 120 & 118 & 210 \\
Number of second line patients & 120 & \\
\hline
\end{tabular}

Table 2 Description of contextual factors

\begin{tabular}{lc}
\hline Variables & Value \\
\hline Type of health facility, n (\%) & $18(15.00)$ \\
Health post & $73(60.83)$ \\
Clinic & $29(24.17)$ \\
Hospital & \\
Having external funding, $\mathrm{n}(\%)$ & $86(71.67)$ \\
No & $34(28.33)$ \\
Yes & \\
Having inpatient bed, n (\%) & $69(57.50)$ \\
No & $51(42.50)$ \\
Yes & \\
Having observation beds, $\mathrm{n}(\%)$ & $88(73.33)$ \\
No & $32(26.67)$ \\
Yes & \\
Having lab, $\mathrm{n}$ (\%) & \\
No & \\
Yes & \\
Share of HIV staff over the total (\%), \\
mean (SD)
\end{tabular}

2,393 clinical records of adults receiving ambulatory ART showed $8.4 \%$ receiving second line treatment. The average unit cost of ART care was US\$297 per adult per year across levels of care (US\$379 at health posts, US\$324 at hospitals, and US\$266 at clinics).

Table 2 describes the characteristics of contextual factors
Table 3 Efficiency scores from DEA by type of facilities

\begin{tabular}{lcc}
\hline Facility & $\mathrm{N}$ & Separate DEA \pm SD \\
\hline Health post & 18 & $0.938 \pm 0.057$ \\
Clinic & 73 & $0.788 \pm 0.077$ \\
Hospital & 29 & $0.797 \pm 0.071$ \\
Total & 120 & $0.812 \pm 0.090$ \\
\hline
\end{tabular}

DEA, data envelopment analysis; SD, standard deviation.

that were used for the regression model. Among 120 facilities, 86 facilities $(71.67 \%)$ did not receive any financial support from donors; $69 \mathrm{did}$ not have inpatient beds; $88 \mathrm{did}$ not have observation beds; and 85 did not have a lab in the facility. On average, staff working on HIV (FTE) accounted for $19.9 \%$ of the total number of staff in the facility, and clinical staff working on HIV represented $51.6 \%$ of total number of FTE staff for HIV. The average HIV prevalence was $17.79 \%$ in the 17 health districts.

\section{Efficiency by type of health facility}

A separate DEA model was used to estimate the efficiency of each type of health facility. DEA score is sensitive to the number of observations in the sample. When DEA is applied to smaller samples, the efficiency score tends to be higher. The average efficiency was $93.8 \%$ among the 18 health posts, $78.8 \%$ for 73 clinics and $79.7 \%$ for the 29 hospitals (Table 3).

\section{Pooled efficiency}

When pooling all three types of health facilities together, the average efficiency is $75.49 \%$ with standard deviation of $7.8 \%$. Figure 1 shows the distribution of the efficiency scores for the 120 health facilities. Most efficiency scores concentrated on the range between $70 \%$ and $85 \%$. 


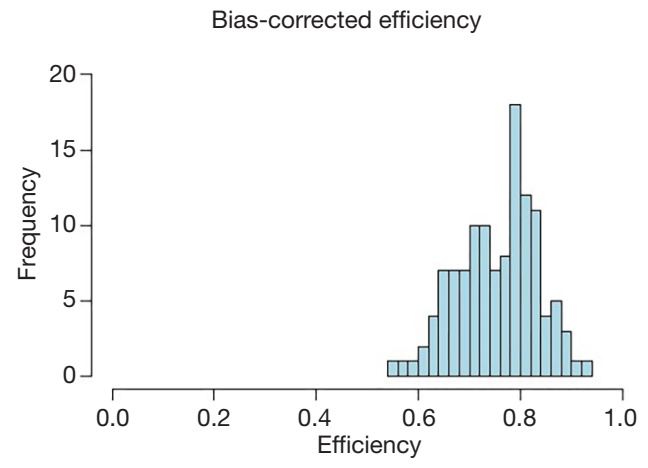

Figure 1 Distribution of efficiency scores among 120 health facilities.

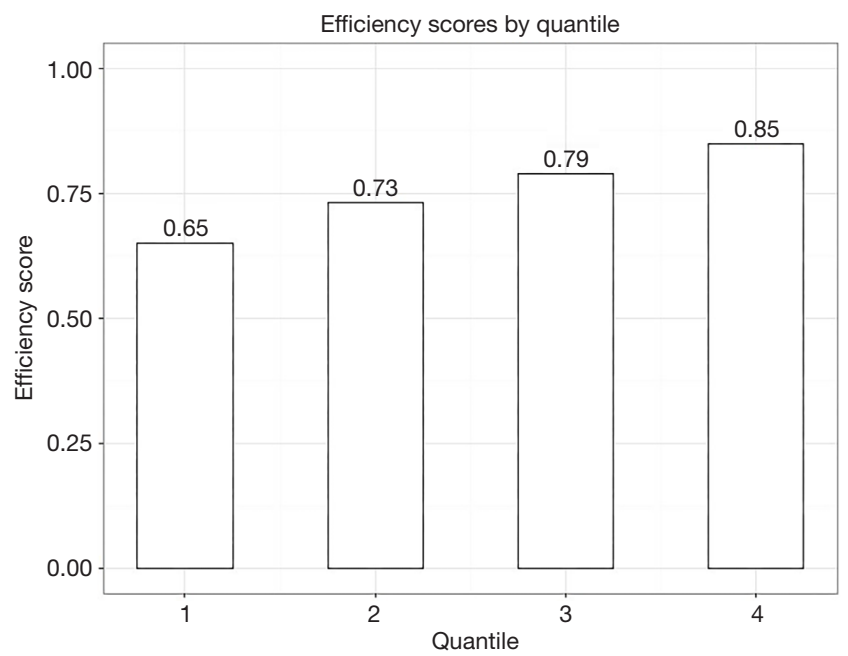

Figure 2 Average efficiency scores by quantile.

Figure 2 shows the average efficiency score by quartiles. The $25 \%$ health facilities with the least performance had an average efficiency score of $65 \%$, suggesting potential savings by optimizing inputs in $35 \%$ and still providing treatment to the same number of the patients. The $25 \%$ health facilities with the best performance had an average score of $85 \%$ and also potential for savings.

\section{Pooled efficiency score by health facility characteristics}

Based on the bivariate analyses, we found that health posts tended to be more efficient than clinics and hospitals (Table 4). The efficiency score for health posts was $78.3 \%$, as compared to $76.0 \%$ for clinics and $72.4 \%$ for hospitals, with $\mathrm{F}$ value of 3.7. The differences were statistically significant $(\mathrm{P}<0.01)$. Health facilities with inpatient beds tended to
Table 4 Efficiency scores by health facility characteristics

\begin{tabular}{|c|c|c|}
\hline Variable & Mean \pm SD (\%) & $\mathrm{F}$ value or $t$ value \\
\hline Type of facility & & $3.7^{\star \star}$ \\
\hline Health post & $78.3 \pm 8.2$ & \\
\hline Clinic & $76.0 \pm 7.8$ & \\
\hline Hospital & $72.4 \pm 6.8$ & \\
\hline External resource & & 1.23 \\
\hline No & $76.0 \pm 7.7$ & \\
\hline Yes & $74.1 \pm 8.0$ & \\
\hline Inpatient bed & & $2.42^{\star *}$ \\
\hline No & $76.9 \pm 8.2$ & \\
\hline Yes & $73.6 \pm 6.8$ & \\
\hline Observation bed & & $2.23^{\star \star}$ \\
\hline No & $76.4 \pm 7.8$ & \\
\hline Yes & $72.8 \pm 7.4$ & \\
\hline Lab in facility & & $3.25^{\star \star \star}$ \\
\hline No & $76.9 \pm 7.7$ & \\
\hline Yes & $72.0 \pm 6.9$ & \\
\hline
\end{tabular}

have a lower efficiency score with $73.6 \%$, as compared to $76.9 \%$ in health facilities without inpatient beds. The difference was statistically significant $(\mathrm{P}<0.01)$. Similarly, health facilities with observation beds and lab tests had a lower efficiency.

\section{Regression analyses}

Table 5 presents determinants of efficiency from the regression models. The first model, excluding facilities with inpatient beds and facilities with laboratories, shows that hospitals tended to have a lower efficiency score than health posts, by $9.7 \%$ points, which is consistent to the results from bivariate analysis. Similarly, health facilities with observation beds had a lower efficiency score than those without, by $5.4 \%$ points. In addition, HIV/AIDS prevalence is positively associated with the efficiency of health facilities in providing ART services. After controlling for inpatient bed and having in-house lab, the coefficients for hospitals was no longer statistically significant. This may suggest that hospitals represent a proxy aggregating a wide variety of factors affecting efficiency. 
Table 5 Determinants of efficiency

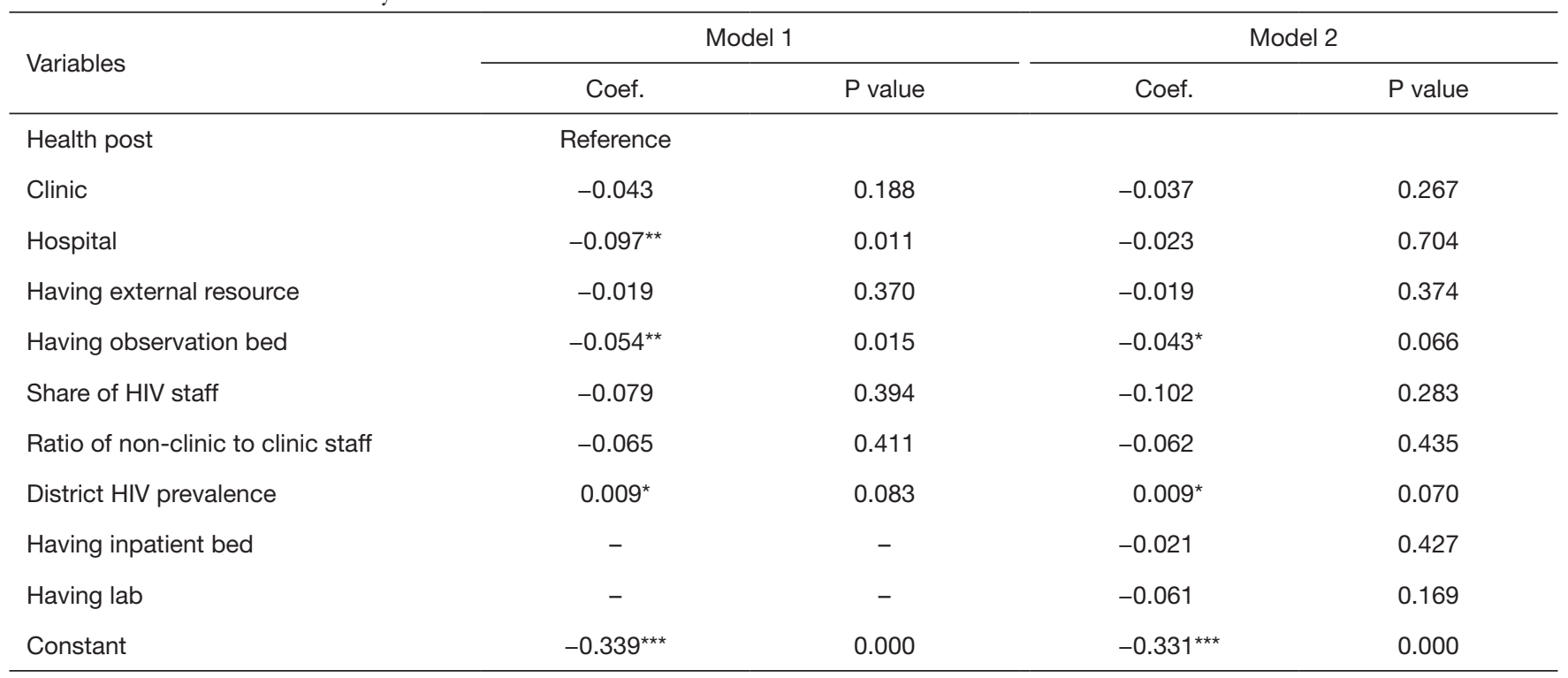

${ }^{*}, \mathrm{P}<0.10$; ${ }^{* \star}, \mathrm{P}<0.05 ;{ }^{* * *}, \mathrm{P}<0.01$. HIV, human immunodeficiency virus.

\section{Discussion}

The overall performance of health facilities in providing ART in Botswana is around $75 \%$, which suggests that improving efficiency would result in $25 \%$ savings if the provision of services is optimized. Meanwhile, the performance of ART services varied substantially, the lowest quartile of health facilities has an average efficiency of $65 \%$. For these facilities, there is a substantial room for improvement. Among the determinants of performance, a hospital facility with observation beds as well as HIV district prevalence are statistically significantly associated with the health facility efficiency.

Our results are consistent with a study carried out in Kenya, Uganda and Zambia (10). In these countries, national averages of efficiency in the provision of ART fell below $50 \%$ and facility-level efficiency markedly varied. Average efficiency scores spanned from $50 \%$ in Uganda to $59 \%$ in Zambia. Under an efficiency improvement scenario, the authors estimated that 459,000 new ART patients could be seen if facilities in these countries reached $80 \%$ efficiency, equivalent to a $40 \%$ increase in new patients.

Efficiency scores observed in Botswana are comparable to another study conducted in Rwanda where health centers scored average efficiency of $78 \%$ (6). The variation in efficiency between health facilities in Botswana is also similar to Rwanda; however, a larger variation in efficiency scores was observed when analyzing large-scale national
HIV programs (5). There are several reasons for smaller variation when compared to large-scale programs. First, this study only focuses on ART treatment, which is quite standard in Botswana. Second, this study does not include other HIV/AIDS services, such as voluntary counseling and testing (VCT) and prevention of mother-to-child transmission (PMTCT) services. Focusing on ART also reduces the heterogeneity of various services in the study, and thus reduces the variation of the performance. Third, in one country, health facilities tend to follow similar treatment guidelines, with similar commodities and resources available for providing ART services. This standardization in the provision of services contributes to a narrowed variation of performance of health facilities compared to national programs.

One of our key findings is that the performance of ART services is lower in hospitals than health posts. Given standard ART protocols for the provision of these services, there is little variation in the cost of medicines and therefore the efficiency lies primarily in the use of human resources and the less standardized use of laboratories. Considering that staff's salaries tend to be higher in hospitals and overall care is more complex at hospitals, usually costs tend to be higher in these facilities, thus resulting in lower performance of hospitals in providing ambulatory services that could be delivered in primary health care settings. This relative low efficiency at hospitals suggests that in order 
to achieve savings, ambulatory services would be more efficiently provided if these services were decentralized to primary health care delivery points. Since most people live in areas closer to health posts, savings in transportation can also be realized by the households. Using health posts for ART services may also help improve the treatment adherence since patients could have better access to health posts and have more regular follow-up visits. In contrast, regularly visiting hospitals is more challenging for patients who live far way. Of course, we should consider the treatment quality between hospital and health post. When considering making use of health posts and clinics for ART services, it is important to strengthen their ART quality, such as through training, to ensure the quality of care is not compromised.

The second interesting finding is that HIV prevalence has a positive impact on the performance of these ART delivery units, which is consistent to prior studies (6). This is perhaps due to the economies of scale. As more HIV/ AIDS patients are concentrated in the same area, more patients seek care in the same facility, so fixed costs could be shared among patients, resulting in higher performance in providing ART. Although health facilities in HIV/AIDS concentrated areas tend to have a higher performance, it should be noted that the facilities should be established strategically, and facilities should not be duplicated within a small area.

Though not directly shown in our study, there are several mechanisms that could be considered to improve the efficiency of health facilities in providing ART services. Task sharing could be one potential mechanism to optimize human resource costs and improve efficiency. Evidence from Ethiopia supports task sharing for ART services, since no significant differences in patient outcomes were found between physician and non-physician clinician-led ART care from 2008 to 2010 (11). Patients seen by nurses or health officers reported higher satisfaction with their ART visits than patients seen by physicians in 2012 (12). In Botswana, the Lay Counselor cadre created in 2001 has helped start patients on ART and lessened burdens on more skilled staff (13). Another study comparing certified nurse prescribers and doctors at a pediatric centre in Botswana, successfully demonstrated comparable performance in HIV management (14). While task shifting and task sharing bear their own costs in the form of training and supportive supervision, lay counselors have been found effective in their roles and satisfactory to patients in Botswana. Task sharing from doctors to nurses and nurses to lay counselors (or similar low-skill cadres), if done with adequate attention to quality, may help reduce unit costs, particularly at health posts, without negatively impacting patient outcomes.

Implementing a differentiated models of care approach is another option for improving efficiency. Differentiated models of care consider patient status to determine their service needs in terms of type, location, provider, and frequency (15). In Malawi, stable adult patients who have been adherent on first line ART 6 months or longer and without side effects are eligible for differential models of care (16). One model, available nationally, is for multimonth prescriptions, in which stable patients reduce their annual number of clinic visits by receiving three or more months of ARVs at a time. Other models involve fewer full-service visits with high level cadres, and communitybased ART groups, where a rotating member picks up ARVs for the entire group. An estimated 69\% of patients are on the multi-month prescriptions model, and annual costs of care for stable patients on any model are $10 \%$ lower than non-model patients. The $2016 \mathrm{WHO}$ guidelines on viral load monitoring also considers differentiated models of care, with stable patients receiving only annual testing after their first year on ART. Sustainability of HIV care in Africa requires viral-load-informed differentiated models of care. Patients with suppressed viral load would require less frequent monitoring, freeing providers to focus on patients with unsuppressed viral load, promoting adherence and allowing timely switching to second-line regimens (17). Utilizing point-of-care viral load testing technology can also help make care more patient-centric by enabling more timely results delivery and improved adherence support (18). By adopting these or similar models of care based on viral load status and targeting the areas of ARV dispensing, service delivery, or lab monitoring, Botswana could more accurately allocate resources based on patient needs, leading to reduced costs and improved efficiency of care, particularly for stable, asymptomatic patients.

Several limitations of this study should be acknowledged. First, for determining efficiency, we focused on facility type and share of resources for HIV/AIDS, and HIV/ AIDS prevalence. We could not include some important variables such as management costs as we did not have such measures in the study. Second, for this study we focused on the public facilities, and did not include private sector in the analysis. Third, we could not control for the quality of care when comparing efficiency by the type of health facilities. Nevertheless, this study demonstrates the efficiency status of public facilities in providing ART services and suggests 
directions for further improving efficiency.

The implementation of the third National Strategic Framework for HIV/AIDS will guide the government's strategic response through 2023 to improve health outcomes for the country's population and represents a stress test for the health system in accelerating access to treatment to nearly 380,000 people living with HIV. Smart health system solutions coupled with innovative service scale-up strategies are critical to sustainably achieving this goal. Our findings demonstrate there is room to increase outputs (number of patients on ART) under the current set of inputs (physical or financial) across all levels of care. Cost savings and efficiency gains in the areas of ARV procurement, routine lab testing, and task shifting among human resources will play an important role in scaling up services. Strengthening these areas and the overall health system will help Botswana continue its impressive progress towards ending the AIDS epidemic as a public health threat by 2030 .

\section{Acknowledgments}

We thank Heather Cogswell and Jose Carlos Gutierrez for their support during the design and data collection process for this study.

Funding: This material is based upon work supported by the United States Agency for International Development under cooperative agreement AID-OAA-A-12-00080. The contents are the responsibility of the authors and do not necessarily reflect the views of USAID or the United States Government.

\section{Footnote}

Provenance and Peer Review: This article was commissioned by the editorial office, Fournal of Hospital Management and Health Policy for the series "Incentives and health system efficiency in low- and middle-income countries". The article has undergone external peer review.

Reporting Checklist: The authors have completed the MDAR reporting checklist. Available at http://dx.doi.org/10.21037/ jhmhp-20-75

Data Sharing Statement: Available at http://dx.doi. org/10.21037/jhmhp-20-75

Conflicts of Interest: All authors have completed the ICMJE uniform disclosure form (available at http://dx.doi. org/10.21037/jhmhp-20-75). The series "Incentives and health system efficiency in low- and middle-income countries" was commissioned by the editorial office without any funding or sponsorship. WZ served as the unpaid Guest Editor of the series and serves as an unpaid editorial board member of Journal of Hospital Management and Health Policy from August 2019 to July 2021. The authors have no other conflicts of interest to declare.

Ethical Statement: The authors are accountable for all aspects of the work in ensuring that questions related to the accuracy or integrity of any part of the work are appropriately investigated and resolved. This study did not involve individual patients; however, it was submitted and approved by the Health Research and Development Committee (HRDC), Ministry of Health, Botswana.

Open Access Statement: This is an Open Access article distributed in accordance with the Creative Commons Attribution-NonCommercial-NoDerivs 4.0 International License (CC BY-NC-ND 4.0), which permits the noncommercial replication and distribution of the article with the strict proviso that no changes or edits are made and the original work is properly cited (including links to both the formal publication through the relevant DOI and the license). See: https://creativecommons.org/licenses/by-nc-nd/4.0/.

\section{References}

1. UNAIDS. Botswana (2019). Available online: https://www. unaids.org/en/regionscountries/countries/botswana

2. Gaolathe T, Wirth KE, Holme MP, et al. Botswana's progress toward achieving the 2020 UNAIDS 90-90-90 antiretroviral therapy and virological suppression goals: a population-based survey. Lancet HIV 2016;3:e221-30.

3. WHO. Botswana: Country Cooperation Strategy at a glance 2014. Available online: http://www.who.int/ countryfocus/cooperation_strategy/ccsbrief_bwa_en.pdf

4. UNICEF. Botswana Budget Brief 2018. Available online: https://www.unicef.org/esaro/UNICEF-Botswana-2018Health-Budget-Brief.pdf

5. Zeng W, Shepard DS, Chilingerian J, et al. How much can we gain from improved efficiency? An examination of performance of national HIV/AIDS programs and its determinants in low- and middle-income countries. BMC Health Serv Res 2012;12:74.

6. Zeng W, Rwiyereka AK, Amico PR, et al. Efficiency of HIV/AIDS health centers and effect of community-based 
health insurance and performance-based financing on HIV/AIDS service delivery in Rwanda. Am J Trop Med Hyg 2014;90:740-6.

7. Murray C, Evans D. Health systems performance assessment: debates, methods and empiricism. Geneva, Switzerland: WHO, 2003.

8. Murray CJ, Frenk J. A framework for assessing the performance of health systems. Bull World Health Organ 2000;78:717-31.

9. Simar L, Wilson PW. Sensitivity analysis of efficiency scores: how to bootstrap in nonparametric frontier models. Management Science 1998;44:49-61.

10. Di Giorgio L, Moses MW, Fullman N, et al. The potential to expand antiretroviral therapy by improving health facility efficiency: evidence from Kenya, Uganda, and Zambia. BMC Med 2016;14:108.

11. Johns B, Asfaw E, Wong W, et al. Assessing the costs and effects of antiretroviral therapy task shifting from physicians to other health professionals in ethiopia. J Acquir Immune Defic Syndr 2014;65:e140-7.

12. Asfaw E, Dominis S, Palen JG, et al. Patient satisfaction with task shifting of antiretroviral services in Ethiopia: implications for universal health coverage. Health Policy

doi: 10.21037/jhmhp-20-75

Cite this article as: Avila C, Zeng W, Cintron C. Efficiency of health facilities providing antiretroviral treatment services in Botswana. J Hosp Manag Health Policy 2020;4:35.
Plan 2014;29 Suppl 2:ii50-8.

13. Ledikwe JH, Kejelepula M, Maupo K, et al. Evaluation of a well-established task-shifting initiative: the lay counselor cadre in Botswana. PLoS One 2013;8:e61601.

14. Monyatsi G, Mullan PC, Phelps BR, et al. HIV management by nurse prescribers compared with doctors at a paediatric centre in Gaborone, Botswana. S Afr Med J 2011;102:34-7.

15. WHO. HIV treatment and care: what's new in service delivery. Fact Sheet. Geneva, Switzerland: WHO, 2015.

16. Clinton Health Access Initiative. Assessing implementation of models of differentiated care for HIV service delivery in Malawi. Boston, MA: Clinton Health Access Initiative, 2016.

17. Working Group on Modelling of Antiretroviral Therapy Monitoring Strategies in Sub-Saharan Africa, Phillips A, Shroufi A, et al. Sustainable HIV treatment in Africa through viral-load-informed differentiated care. Nature 2015;528:S68-76.

18. Cogswell HA, Ohadi E, Avila C. Viral-load point-of-care technologies to achieve an AIDS-free generation. Future Microbiol 2016;11:5-9. 